\title{
RDF as a Data Model
}

\section{(Invited Talk)}

\author{
Claudio Gutiérrez * \\ Center for Web Research, Computer Science Department, Universidad de Chile, \\ Blanco Encalada 2120, 3er piso, Santiago, Chile. cgutierr@dcc.uchile.cl
}

\section{Abstract}

The Resource Description Framework (RDF) is the W3C recommendation language for representing metadata about Web resources. It is the basic data layer of the Semantic Web. The original design was influenced by the Web, library, $\mathrm{XML}$ and Knowledge representation communities. The driving idea was a language to represent information in a minimally constraining and flexible way. It turns out that the impact of the proposal goes far beyond the initial goal, particularly as a model for representing information with a graph-like structure.

In the first half of the talk we will review RDF as a database model, that is, from a data management perspective. We will compare it with two data models developed by the database community which have strong similarities with RDF, namely, the semistructured and the graph data models. We will focus the comparison on data structures and query languages.

In the second half of the talk, we will discuss some of the challenges posed by RDF to the Computer Science Theory Community:

1. RDF as data model: Database or knowledge base?

2. Abstract model for RDF: What is a good foundation?

3. Concrete -real life- RDF data: What are the interesting fragments?

4. Theoretical novelties of the RDF data model: Are there any?

5. RDF Query Language: Can the database experience be of any help?

6. Infrastructure for large-scale evaluation of data management methodologies and tools for RDF: Waiting for something?

7. Storing, Indexing, Integrity Constraints, Visualization et al.: Theory is required.

* The speaker acknowledges the support of Millennium Nucleus Center for Web Research, Grant P04-067-F, Mideplan, Chile.

Please use the following format when citing this chapter:

Gutiérrez, C., 2006, in International Federation for Information Processing, Volume 209, Fourth IFIP International Conference on Theoretical Computer Science-TCS 2006, eds. Navarro, G., Bertossi, L., Kohayakwa, Y., (Boston: Springer), p. 7. 